\title{
Helicobacter pylori prevalence in dyspeptic patients in the Eastern Cape province - race and disease status
}

\author{
N F Tanih, B I Okeleye, L M Ndip, A M Clarke, N Naidoo, N Mkwetshana, E Green, R N Ndip
}

Objectives. We examined Helicobacter pylori infection in patients with gastric-related morbidities at Livingstone Hospital, Port Elizabeth, to determine the prevalence and risk factors for infection according to race, endoscopic diagnosis, age and sex.

Methods. Gastric biopsies were collected from 254 consecutive patients and $H$. pylori was isolated on Columbia agar base supplemented with 7\% sheep's blood and Skirrow's supplement containing trimethoprim $(2.5 \mathrm{mg})$, vancomycin $(5 \mathrm{mg})$ and cefsulodin $(2.5 \mathrm{mg})$. Amphotericin $(2.5 \mathrm{mg})$ was added to the medium. Recovered isolates were identified following standard microbiology and biochemical techniques. Presumptive isolates were further confirmed by polymerase chain reaction (PCR) targeting the $\operatorname{glm} M$ gene. Fisher's exact test was used to assess the univariate association between $H$. pylori infection and the possible risk factors. Odds ratios (ORs) and corresponding 95\% confidence intervals (CIs) were calculated to measure the strength of association, using EPI INFO 3.41 software. $p$-values $<0.05$ were required for significance.

Results. The overall prevalence of $H$. pylori was $66.1 \%$ (168/254). Of the 168 positive subjects, $H$. pylori prevalence was highest in patients with non-ulcer dyspepsia (NUD) $(32.7 \%$; 55/168), and lowest $(0 \% ; 0 / 168)$ in those with atypical oesophageal reflux disease and gastroduodenitis, respectively. The prevalence of infection was highest among coloureds $(68.4 \% ; 89 / 130)$ and lowest in whites (59.5\%; 25/42). Prevalence increased with age.

Conclusion. The prevalence of $H$. pylori is high in dyspeptic patients in Eastern Cape province. Gender, antibiotic treatment and alcohol consumption may be risk factors for infection. These findings are of clinical and epidemiological significance.

S Afr Med J 2010; 100: 734-737.
Helicobacter pylori is the principal species of the genus Helicobacter that inhabits the gastric mucosa of the human stomach. It chronically infects billions of people worldwide, ${ }^{1-3}$ and is responsible for one of the most frequent chronic bacterial infections involving more than $50 \%$ of the world's population. ${ }^{4} \mathrm{H}$. pylori is one of the most genetically diverse of bacterial species, and is a major cause of at least $90 \%$ of duodenal ulcers (DUs), 70\% of gastric ulcers (GUs), non-ulcer dyspepsia (NUD) and gastro-oesophageal reflux disease (GORD). ${ }^{5,6}$ It plays a role in adenocarcinoma of the distal stomach, mucosaassociated lymphoid tissue lymphoma (MALT) and primary gastric non-Hodgkin's lymphoma, in many societies. ${ }^{3,7} \mathrm{H}$. pylori's ability to adhere, invade and evade host defences and cause tissue damage is largely due to its production of colonisation and virulence factors. ${ }^{8}$

The prevalence of $H$. pylori infection increases with advancing age and is higher in developing countries and among low socio-economic populations, probably owing to conditions that favour the infection,

Department of Biochemistry and Microbiology, University of Fort Hare, Alice, E Cape N F Tanih, MSc

B I Okeleye, BSc (Hons)

A M Clarke, $\mathrm{PhD}$

N Mkwetshana, $\mathrm{PhD}$

E Green, MSc

R N Ndip, $\mathrm{PhD}$

Department of Biochemistry and Microbiology, Faculty of Science, University of Buea, Cameroon

R N Ndip, $\mathrm{PhD}$

L M Ndip, $\mathrm{PhD}$

Department of Gastroenterology, Livingstone Hospital, Port Elizabeth, E Cape N Naidoo, MB ChB, FCP (SA) such as poor hygiene, crowded living conditions, and inadequate or no sanitation. ${ }^{1,9-11}$

Although the geographical and socio-demographic prevalence of human infection by $H$. pylori varies, ${ }^{1,11}$ prevalence does not parallel the incidence of morbidity caused by the infection. In Africa, for example, $H$. pylori infection is common ${ }^{1,2,10,11}$ and is the main cause of at least $90 \%$ of duodenal ulcers and $70 \%$ of gastric ulcers on the continent. ${ }^{2,10}$ Involvement of $H$. pylori in gastric patients in Cameroon $^{2}$ revealed a similar pattern to that recorded among Ghanian patients ${ }^{12}$ with dyspeptic symptoms and in South African patients with NUD. ${ }^{13}$

The prevalence of $H$. pylori infection differs in relation to ethnic groups, races and economic conditions. ${ }^{14} \mathrm{H}$. pylori infection seems to be common in South Africa, as might be expected in developing countries. ${ }^{15,16}$ Although studies have reported the prevalence of the organism in the country, most adopted a sero-prevalence approach, which does not provide more information other than the presence of antibodies, which are poor markers of active infection. In Cape Town, a prevalence of $63 \%$ of the pathogen in NUD patients based on histology reports was documented, ${ }^{13}$ and a prevalence of $81 \%$ in NUD patients based on serology testing. ${ }^{17}$

Published data on the prevalence of the organism in dyspeptic patients in the Eastern Cape province, which is predominantly rural with poor sanitation and household hygiene (factors that favour the spread of $H$. pylori), are lacking. Therefore, investigating its prevalence in the province based on race and disease profile is important in forming the basis of future clinical and epidemiological studies.

\section{Methods Subjects}

We evaluated 254 consecutive patients referred for endoscopy at Livingstone Hospital, Port Elizabeth, between May and December 2008. The nature and purpose of the study was explained to the patients until they fully understood. We enrolled only patients who gave informed consent; who had not received treatment with broad- 
spectrum antibiotics, non-steroidal anti-inflammatory drugs or proton pump inhibitors in the previous 3 months; and who did not have a history of dysphagia, gastric surgery or upper gastro-intestinal bleeding. The study was approved by the institutional review board of the University of Fort Hare and the Eastern Cape Department of Health (Protocol number EcDoH-Res 0002).

\section{Endoscopy, questionnaire and culture for $\boldsymbol{H}$. pylori}

All patients underwent a complete physical examination and history was taken by a resident gastro-enterologist. Endoscopic diagnoses were made by a single experienced endoscopist to rule out observer bias. The diagnoses were made on the macroscopic appearance of obviously inflamed mucosa at endoscopy, i.e. hyperaemic oedematous mucosa sometimes with contact bleeding to delineate the different clinical conditions associated with infection. Race, smoking, alcohol intake and demographic data were recorded. Some patients had previously undergone $H$. pylori eradication therapy.

Two antral and corpus biopsy specimens each were obtained at endoscopy for a total of 1016 specimens, which were immediately placed in sterile bijou bottles containing $0.2 \mathrm{~g} / \mathrm{l}$ of cysteine and $20 \%$ glycerol in brain-heart infusion (BHI) broth and transported in ice to the laboratory within 2 hours of collection for culture and characterisation as previously reported. ${ }^{2}$ Presumptive isolates were confirmed by polymerase chain reaction (PCR) targeting the glmM gene as previously reported. ${ }^{18} \mathrm{~A}$ reference strain of $H$. pylori (NCTC 11638) was included as a positive control. Confirmed isolates were suspended in $20 \%$ glycerol and stored at $-80^{\circ} \mathrm{C}$ in a freezer (Sanyo, Japan) for future experiments.

\section{Statistical analysis}

An SPSS program was used to determine Pearson's chi-square test and Fisher's exact test. Fisher's exact test was used to assess the univariate association between $H$. pylori infection and the possible risk factors. Odds ratio (OR) and the corresponding 95\% confidence interval (CI) were calculated to measure the strength of association using EPI INFO 3.41 package (Centers for Disease Control and Prevention, Atlanta, GA, USA). $p$-values $<0.05$ were required for significance.

\section{Results}

A total of 254 patients were enrolled. Their mean age was $44.5 \pm 15.7$ years (range 5 - 93 years), and $83.1 \%(211 / 254)$ were older than 35 years. There were 90 males and 164 females (male/female ratio $1: 1.8)$.

Endoscopy was performed on all 254 enrolled patients. Of the abnormal findings, NUD was the most common (33.8\%; 86/254), followed by GORD (17.7\%; 45/254), DU (15.7\%; 40/254), and GU (9.4\%; 24/254) (Table I).

Of the 254 subjects, 168 had $H$. pylori-positive culture, giving an overall prevalence of $66.1 \%$. A total of $592 \mathrm{H}$. pylori strains were obtained from the 1016 biopsy specimens (508 antral and 508 corpus). This was confirmed by PCR as we obtained the expected 1.1 kilobase for the organism. The mean incubation time was 4 days. The percentage positivity for antrum was $61.0 \%$ (310/508), and for corpus $55.5 \%(282 / 508)$.

Of our 168 positive subjects, $H$. pylori prevalence was highest in patients with NUD $(32.7 \% ; 55 / 168)$ and lowest $(0 \% ; 0 / 168)$ in those with atypical oesophageal reflux disease and gastroduodenitis respectively (Table I). Also, of the 254 enrolled subjects, the prevalence of $H$. pylori in relation to endoscopic findings was highest (100\%) in patients with duodenitis (1/1), gastric cancer (GC) (4/4) and gastric erosion (5/5), as they were all positive for the organism. However, patients with gastritis $(6 / 8 ; 75 \%), \mathrm{GU}(17 / 24 ; 70.8 \%)$, DU (26/40 $65 \%)$, NUD (55/85; 64.7\%), and GORD $(29 / 45 ; 64.4 \%)$ also had high prevalence rates for the organism (Table I).

Of the 254 enrolled patients, 82 (32.2\%) were black, 130 (51.1\%) were coloured and $42(16.5 \%)$ were white. H. pylori prevalence was highest among the coloureds $(68.4 \% ; 89 / 130)$, followed by blacks $(65.8 \% ; 54 / 82)$ and whites $(59.5 \% ; 25 / 42)$. The prevalence of the organism by gender in these three racial groups was highest among black females $(71.6 \%$; 38/53), followed by whites $(68.1 \%$; $15 / 22)$ and coloureds $(67.7 \% ; 61 / 90)$. In males, the prevalence was highest among the coloured group $(70 \% ; 28 / 40)$, followed by the black $(55.1 \% ; 16 / 29)$ and white $(50 \% ; 10 / 20)$ groups. Differences between the racial groups, sex and age were, however, not statistically significant $(p>0.05)$.

The prevalence of $H$. pylori infection increased with age (Table II). Regarding sex, 114 (69.5\%) of the 164 enrolled females were positive, while $54(60 \%)$ of the 90 males were positive.

The prevalence of $H$. pylori related to alcohol consumption and smoking, respectively, was also investigated. Of the 168 subjects positive for $H$. pylori, 71 (42.3\%) were smokers and 46/86 (53.5\%) non-smokers; and 60/168 (35.7\%) positive subjects drank alcohol, while $28 / 86$ (32.6\%) did not (Table II).

\section{Discussion}

The high prevalence in Africa of human infection by H. pylori indicates that effective public health interventions should be developed. The variations in its prevalence between and among populations could indicate that factors including age, cultural background, genetic predisposition, socio-economic status and environmental conditions all play a role in the acquisition and transmission of the organism. ${ }^{11,12}$ Within countries, the prevalence among more affluent urban populations and poor rural populations can vary widely. ${ }^{11,12}$

All the dyspeptic patients investigated in this study presented with signs of gastric-related morbidities at the endoscopic unit of Livingstone Hospital in Port Elizabeth. We used cultures (confirmed by molecular techniques) to investigate the prevalence of $H$. pylori infection among dyspeptic patients and also to delineate the risk factors for infection among ethnic groups and races related to other demographic characteristics. Although some authors have dismissed culture as the gold standard for $H$. pylori diagnosis, others have proved it useful in large-scale epidemiological studies and in laboratories with limited resources in the developing world., ${ }^{2,19}$ Culture also enabled us to determine the susceptibility patterns of our isolates to guide empiric treatment. ${ }^{20}$

Our overall prevalence finding of $66.1 \%(168 / 254)$ is similar to studies in Cameroon, Cote d'Ivoire, Egypt, Ghana, Kenya, Korea, Malawi, Nigeria, South Africa, Zaire and Zimbabwe (60 $94 \%) .^{2,9,12,21}$ Kidd et al. ${ }^{22}$ also reported that endoscopic studies in dyspeptic individuals in Africa revealed the presence of the organism in most subjects. Previous South African studies ${ }^{10,13,17}$ had equally incriminated this organism in cases of duodenal and gastric ulcers.

By endoscopic diagnosis, H. pylori prevalence was highest (100\%) among patients with duodenitis (1/1), GC (4/4) and gastric erosion $(5 / 5)$. Our reported prevalence of $100 \%(4 / 4)$ in patients with GC by endoscopic diagnosis and $H$. pylori prevalence is very high compared with other studies in Africa, and differs from the findings of Uemura et al. ${ }^{23}$ (considering only those with GC) who reported GC in $2.9 \%$ of $H$. pylori-infected and none of their uninfected patients. However, the possible reason for our unusually high prevalence could be linked to the different sample sizes used in these studies. Only 4 of our 


\section{Table I. Gastro-duodenal pathologies and prevalence of $H$. pylori infection in 254 patients referred for endoscopy}

\begin{tabular}{lccc}
\hline & & \multicolumn{2}{c}{ H. pylori positive (\%) } \\
\cline { 2 - 4 } Endoscopic categories & No. of cases (\%) & Endoscopic diagnosis & Study subjects \\
\hline Non-ulcer dyspepsia & $85(33.4)$ & $55(64.7)$ & $55(32.7)$ \\
GORD & $45(17.7)$ & $29(64.4)$ & $29(17.2)$ \\
Duodenal ulcer & $40(15.7)$ & $26(65)$ & $26(15.4)$ \\
Gastric ulcer & $24(9.4)$ & $17(70.8)$ & $17(10.1)$ \\
Gastritis & $8(3.1)$ & $6(75)$ & $6(3.5)$ \\
Duodenitis & $1(0.3)$ & $1(100)$ & $1(0.5)$ \\
Gastroduodenitis & $2(0.7)$ & $0(00)$ & $0(00)$ \\
Gastric cancer & $4(1.5)$ & $4(100)$ & $4(2.3)$ \\
Gastric erosion & $5(1.9)$ & $5(100)$ & $5(2.9)$ \\
Antral gastritis & $2(0.7)$ & $1(50)$ & $1(0.5)$ \\
Atypical ORD & $1(0.3)$ & $0(00)$ & $0(00)$ \\
Others & $37(14.5)$ & $24(64.9)$ & $24(14.2)$ \\
Total & 254 & 168 & \\
GoRD=gastro-oesopharyngeal reflux disease; ORD=oesopharyngeal reflux disease. & & \\
\hline
\end{tabular}

Table II. Association between epidemiological risk factors and $H$. pylori infection (univariate analysis)

\begin{tabular}{|c|c|c|c|c|c|c|}
\hline $\begin{array}{l}\text { Variables and } \\
\text { categories }\end{array}$ & $\begin{array}{l}\text { No. of } \\
\text { cases (\%) }\end{array}$ & $\begin{array}{l}\text { H. pylori } \\
\text { +ve }(\%)\end{array}$ & $\begin{array}{l}\text { H. pylori } \\
\text {-ve (\%) }\end{array}$ & OR $(95 \%$ CI $)$ & $\chi^{2}$ test & $p$-value \\
\hline \multicolumn{7}{|l|}{ Age (yrs) } \\
\hline $15-24$ & $10(4)$ & $8(80)$ & $2(20)$ & $1.00(0.06-17.08)$ & 0.31 & 1.0 \\
\hline $25-34$ & $33(13)$ & $21(63.6)$ & $12(36.4)$ & $0.44(0.04-2.78)$ & 0.94 & 0.5 \\
\hline $35-44$ & $40(16)$ & $25(62.5)$ & $15(37.5)$ & $0.42(0.04-2.65)$ & 0.45 & 0.5 \\
\hline $45-54$ & $70(28)$ & $51(73)$ & $19(27)$ & $0.67(0.06-3.82)$ & 0.01 & 1.0 \\
\hline $55-64$ & $47(18)$ & $35(74.5)$ & $12(25.5)$ & $0.73(0.07-4.45)$ & 0.00 & 1.0 \\
\hline$>65$ & $54(21)$ & $28(51.8)$ & $26(48.2)$ & $0.27(0.03-1.55)$ & 1.69 & 0.2 \\
\hline \multicolumn{7}{|l|}{ Gender } \\
\hline Male & $90(35.4)$ & $54(60)$ & $36(40)$ & 1 & & \\
\hline Female & $164(65.6)$ & $114(69.5)$ & $50(30.5)$ & $1.45(0.82-2.57)$ & 1.47 & 0.2 \\
\hline \multicolumn{7}{|c|}{ Antibiotic treatment } \\
\hline Yes & $16(6.3)$ & $14(87.5)$ & $2(12.5)$ & $3.82(0.84-35.27)$ & 2.53 & 0.11 \\
\hline No & $238(93.7)$ & $154(64.7)$ & $84(35.3)$ & 1 & & \\
\hline \multicolumn{7}{|l|}{ Smoking } \\
\hline Yes & $168(66)$ & $71(42.3)$ & $97(57.7)$ & $0.64(0.36-1.11)$ & 2.45 & 0.1 \\
\hline No & $86(34)$ & $46(53.5)$ & $40(46.5)$ & 1 & & \\
\hline \multicolumn{7}{|c|}{ Alcohol consumption } \\
\hline Yes & $168(66)$ & $60(35.7)$ & $108(64.3)$ & $1.15(0.64-2.07)$ & 0.13 & 0.7 \\
\hline No & $86(34)$ & $28(32.6)$ & $58(67.4)$ & 1 & & \\
\hline
\end{tabular}

254 patients had GC, giving an overall prevalence of $2.38 \%$ which, considering the overall study subjects, is in line with the findings of Uemura et al..$^{23}$

Another consideration is that South Africa has a heterogeneous population including whites whose ancestral background is in the Western world where GC is high; ${ }^{23}$ our 4 patients with GC were white. GC is a terminal condition for $H$. pylori-positive patients; hence, a patient once identified as having GC will invariably have the infection. Also, GC develops in persons with $H$. pylori but not in uninfected persons. ${ }^{23}$

Patients with gastritis $(75 \% ; 6 / 8)$, GU $(70.8 \%$; $17 / 24)$, DU (65\%; $26 / 40)$, NUD (64.7\%; 55/85), and GORD (64\%; 29/45) also had high prevalence rates of the organism, which corroborates the findings of others ${ }^{7,21}$ who reported either one or two of the above clinical conditions as the most common endoscopic findings in $\mathrm{H}$. pylori infection. It has been suggested that up to $95 \%$ of DUs and $70 \%$ of GUs are attributable to infection by this pathogen, and most cases occur in middle-aged subjects. ${ }^{2,10}$

Significantly, our results are consistent with previous studies in South Africa. ${ }^{13,17}$ Louw et al. ${ }^{13}$ documented an overall incidence of $\mathrm{H}$. pylori infection in $80 \%$ of GU and $95 \%$ of DU patients, and O'Keefe et al. ${ }^{17}$ reported overall $H$. pylori prevalence of $80 \%$, with prevalence rates of $78 \%, 81 \%$, and $81 \%$ in patients with GC, peptic ulcer (PU) and NUD, respectively.

The prevalence of $H$. pylori infection was highest among the coloured $(68.4 \%-87 / 130)$ and black subjects $(65.8 \%-54 / 82)$ and lowest in whites $(59.5 \%-25 / 42)$. There was no statistically significant difference between the races, in contrast with the findings of Louw et $a l .{ }^{10}$ in the Western Cape province, where they demonstrated a significant difference: a prevalence of $40 \%$ in white and $71 \%$ in 
coloured patients. However, both our studies reported a higher prevalence among coloureds than whites. The high prevalence of $H$. pylori infection among coloured and black South Africans compared with whites could partly be explained by other known risk factors for H. pylori, particularly socio-economic determinants such as lower income, lower educational levels, and household crowding., ${ }^{910}$

Females - particularly blacks - had a higher prevalence (71.6\%) than males $(55.1 \%)$. Some studies have found a higher prevalence among women, but many have found no gender-based difference. ${ }^{24}$ It is possible that the risk of acquisition does not differ between the sexes. We speculate that females are more likely to have infections (such as genito-urinary infections) that are eradicated by antimicrobials such as metronidazole, which is used for other micro-organisms and commonly selects for $H$. pylori-resistant strains. ${ }^{2}$ Although we tried to ensure that our subjects had not been on antimicrobials for 3 preceding months, their use cannot be ruled out.

We also observed that $H$. pylori infection prevalence increased with age, similarly to the results of Chong et al. ${ }^{25}$ The organism is ubiquitous, with acquisition in childhood being the rule. In our study, alcohol consumption and smoking were not significantly associated with $H$. pylori infection ( $p>0.05$ ), although the OR (OR 1.15; 95\% CI 0.64 - 2.07) indicates that alcohol consumption may be a risk factor for H. pylori infection. H. pylori prevalence among alcohol consumers has been suggested to be lower than that in non-drinkers because alcohol may kill the organism in the stomach; however, this remains a matter for speculation. ${ }^{14}$

In conclusion, the prevalence of $H$. pylori is high in dyspeptic patients in Eastern Cape province. The ORs indicate that gender, antibiotic treatment and alcohol consumption may be risk factors for infection. These findings have clinical and epidemiological significance and therefore call for more comprehensive studies involving molecular epidemiology and genetic typing methods, to further elucidate the situation.

This study received financial support from the National Research Foundation (NRF) of South Africa (grant reference 69815) and the Govan Mbeki Research and Development Centre, University of Fort Hare. We are also grateful to the staff of the GIT Unit, Livingstone Hospital, Port Elizabeth and Mr B Clarke for technical assistance.

\section{References}

1. Asrat D, Nilsson I, Mengistu Y, et al. Prevalence of Helicobacter pylori infection among adult dyspeptic patients in Ethiopia. Ann Trop Med Parasitol 2004;98(2):181-189.

2. Ndip RN, Malange TAE, Ojongokpoko JEA, et al. Helicobacter pylori isolates recovered from gastric biopsies of patients with gastro-duodenal pathologies in Cameroon: current status of antibiogram. Trop Med Int Health 2008;13(6):848-854

3. Carrilho C, Modcoicar P, Cunha L, et al. Prevalence of Helicobacter pylori infection, chronic gastritis, and intestinal metaplasia in Mozambican dyspeptic patients. Virchows Archiv 2009;454(2):153-160.

4. Go MF. Review article: natural history and epidemiology of Helicobacter pylori infection. Aliment Pharmacol Ther 2002;1:3-15.

5. Henriksen TH. Peptic ulcer disease is strongly associated with Helicobacter pylori in East, West, Central and South Africa. Scand J Gastroenterol 2001;36:561-564.

6. Figueiredo C, Machado JC, Yamaoka Y. Helicobacter pylori infection. Helicobacter 2005;10:14-23.

7. Matsuhisa TM, Yamada NY, Kato SK, Matsukura NM. Helicobacter pylori infection, mucosal atrophy and intestinal metaplasia in Asian populations: a comparative study in age-, gender- and endoscopic diagnosis-matched subjects. Helicobacter 2003;8(1):29-35.

8. Horiuchi T, Ohkusa T, Watanabe M, Kobayashi D, Miwa H, Eishi Y. Helicobacter pylori DNA in drinking water in Japan. J Microbiol Immunol 2001;45 (7):515-519.

9. Dube C, Nkosi TC, Clarke AM, Mkwetshana N, Green E, Ndip RN. Helicobacter pylori in an asymptomatic population of Eastern Cape Province, South Africa: Public health implication. Rev Environ Health 2009;24 (3):249-255

10. Louw JA, Jaskiewicz K, Girdwood AH, et al. Helicobacter pylori prevalence in non-ulcer dyspepsiaethnic and socio-economic differences. S Afr Med J 1993;83(3):169-171.

11. Ndip RN, Malange AE, Akoachere JFT, Mackay WG, Titanji VPK, Weaver LT. Helicobacter antigen in faeces of asymptomatic children in the Buea and Limbe health districts of Cameroon: a pilot study. Trop Med Int Health 2004;9(9):1036-1040.

12. Baako BN, Darko R. Incidence of Helicobacter pylori infection in Ghanian patients with dyspeptic symptoms referred for upper gastrointestinal endoscopy. West Afr J Med 1996;15(4):223-227.

13. Louw JA, Falck C, van Rusburg C, Adams G, Marks IN. Distribution of Helicobacter pylori colonisation and associated gastric inflammatory changes: differences between patients with duodenal and gastric ulcers. J Clin Pathol 1993;46(8):754-756.

14. Iso N, Matsuhisa T, Shimizu K. Helicobacter pylori infection among patients visiting a clinic in Kasama City. J Nippon Med Sch 2005;72(6):341-354.

15. Pelser HH, Househam KC, Joubert G, van der Linde G, Kraaj P, Meinardi M. Prevalence of Helicobacter pylori antibodies in children in Bloemfontein, South Africa. J Pediatr Gastroenterol Nutr 1997;24(2): $135-139$

16. Mosane TW, Malope BI, Ratshikhopha ME, Hiss DC, Sitas F. Seroprevalence of Helicobacter pylori IgG antibodies in South African mothers and their children. Eur J Gastroenterol Hepatol 2004;16(1) $113-114$

17. O'Keefe SJ, Salvador B, Nainkin J, Majiki S, Stevens H, Atherstone A. Empiric treatment based on Helicobacter pylori serology cannot substitute for early endoscopy in the management of dyspeptic rural black Africans. S Afr Med J 2000;90(11):1129-1135.

18. Burucoa C, Lhomme V, Fauchere JL. Performance criteria of DNA fingerprinting methods for typing of Helicobacter pylori isolates: Experimental results and meta-analysis. J Clin Microbiol 1999;37(12): 4071-4080.

19. Mégraud F, Lehn N, Lind T, et al. Antimicrobial susceptibility testing of Helicobacter pylori in a large multicenter trial: the MACH 2 study. Antimicrob Agents Chemother 1999;43(11):2747-2752.

20. Tanih NF, Okeleye BI, Naidoo N, et al. Marked susceptibility of South African Helicobacter pylor strains to ciprofloxacin and amoxicillin: Clinical implication. S Afr Med J 2009;100(1):49-52.

21. Kidd M, Louw JA, Mark NI. Helicobacter pylori in Africa: observation on an 'enigma within an enigma. J Gastroenterol Hepatol 1999;14:851-858.

22. Kidd M, Peek RM, Lastovica AJ, Israel DA, Kummer AF, Louw JA. Analysis of iceA genotypes in South African Helicobacter pylori strains and relationship to clinically significant disease. Gut 2001, 49(5):629-635.

23. Uemura N, Okamoto S, Yamamoto S, et al. Helicobacter pylori infection and the development of gastric cancer. N Engl I Med 2001;345:784-789.

24. Replogle ML, Glaser SL, Hiatt RA, Parsonnet J. Biologic sex as a risk factor for Helicobacter pylon infection in healthy young adults. Am J Epidemiol 1995;142(8):856-863

25. Chong VH, Lim KC, Rajendran N. Prevalence of active Helicobacter pylori infection among dyspeptic patients referred for endoscopy in Brunei Darussalam. Singapore Med J 2008;49:42-46.

\section{LIVE LAPAROSCOPIC PAEDIATRIC WORKSHOP 12 - 14 November 2010}

The Division of Paediatric Surgery will be hosting a Live Laparoscopic Paediatric Workshop at Chris Hani Baragwanath Hospital on 12 - 14 November 2010. The international expert will be Dr Joe Curry, the lead paediatric surgeon at Great Ormond Street Hospital, London.

In addition to the surgical programme, a free paper session will be held on Sunday 14 November, from which a dedicated issue of the SA Journal of Surgery will be published in 2011. 